\title{
THE $p$-ADIC LOG GAMMA FUNCTION AND p-ADIC EULER CONSTANTS ${ }^{1}$.
}

BY

\author{
JACK DIAMOND
}

\begin{abstract}
We define $G_{p}$, a $p$-adic analog of the classical log gamma function and show it satisfies relations similar to the standard formulas for $\log$ gamma. We also define $p$-adic Euler constants and use them to obtain results on $G_{p}^{\prime}$ and on the logarithmic derivative of Morita's $\Gamma_{p}$.
\end{abstract}

1. Introduction. Leopoldt and Kubota defined $p$-adic $L$-functions by summing a function of two variables with respect to one of the variables. We present a general theorem on this technique and then use it to define $G_{p}$, a $p$-adic analog of the classical $\log \Gamma$ function. We work with $\log \Gamma$ rather than $\Gamma$ because the only continuous $p$-adic function defined on a subset of $\Omega_{p}$ and satisfying $f(x+1)=x f(x)$ is the zero function. It is possible to construct an analog of $\Gamma$ by modifying the functional equation (see Morita [7]), but then we do not have close analogs of the standard formulas for $\Gamma$ or $\log \Gamma$. For $G_{p}$, which is not the log of Morita's gamma function, we have the functional equation, an extension theorem, the Stirling series, the Gauss multiplication theorem, a power series, certain "Laurent" series and a formula due to Gauss which is valid for $G_{p}^{\prime}$ at rational points.

This last formula was discussed by Lehmer in [6], where he defined Euler constants for arithmetic progressions. We define $p$-adic Euler constants and present a proof of Gauss' theorem which is valid in both the $p$-adic and complex systems. We also apply the results on Euler constants to obtain a finite expression for the logarithmic derivative of Morita's $p$-adic gamma function at certain rational values in its domain.

2. Notation and definitions. We will use $Q, Q_{p}, Z, Z_{p}, C$ and $\Omega_{p}$ for, respectively, the field of rational numbers, the $p$-adic completion of $Q$, the ring of rational integers, the $p$-adic completion of $Z$ in $Q_{p}$, the field of complex numbers and the completion of the algebraic closure of $Q_{p} . B_{n}$ will be the $n$th Bernoulli number defined by $t e^{t} /\left(e^{t}-1\right) . \nu$ will be the $p$-adic valuation on

Received by the editors April 12, 1976.

AMS (MOS) subject classifications (1970). Primary 12B40; Secondary 33A15.

Key words and phrases. Log gamma function, Euler constants, $p$-adic functions.

${ }^{1}$ This paper is part of the author's Ph.D. dissertation, submitted to Princeton University in July, 1975.

- American Mathematical Society 1977 
$\Omega_{p}$ with $\nu(p)=1$ and ||$_{p}$ will be the absolute value on $\Omega_{p}$ with $|p|_{p}=p^{-1}$. We will use boldface letters to indicate $r$-tuples.

A polydisc about $\mathbf{c} \in \Omega_{p}^{r}$ is a set of the form

$$
\left\{\left(x_{1}, \ldots, x_{r}\right):\left|x_{i}-c_{i}\right|_{p} \leqslant \rho_{i}, i=1,2, \ldots, r\right\}
$$

where $\mathbf{c}=\left(c_{1}, \ldots, c_{r}\right)$ and all $\rho_{i}>0 .\left(\rho_{1}, \ldots, \rho_{r}\right)$ is called the radius of the polydisc. a and $\mathbf{M}$ will denote $\left(a_{1}, \ldots, a_{r}\right)$ and $\left(M_{1}, \ldots, M_{r}\right)$, respectively.

We call a function defined on a subset of $\Omega_{p}^{r}$ holomorphic if it can be represented by a single power series and locally holomorphic if at each point in the domain we can represent the function by a power series on some polydisc containing the point.

The author wishes to express his gratitude to Professor Bernard Dwork for many helpful discussions on this material.

3. $p$-adic sums. We begin by considering sums of the type used by Leopoldt and Kubota [5]. The first theorem is a generalization of a result in [2, p. 309].

THEOREM 1. Suppose we have rational integers $a_{i}, b_{i}, M_{i}$ with $a_{i}>0, b_{i}>$ $1, M_{i} \geqslant 1$ for $i=1,2, \ldots, r$. Let $R$ be an open set in $\Omega_{p}^{r}$ with $\mathbf{a}+\mathbf{M} Z_{p} \subset R$. $B$ is a Banach space over $\Omega_{p}$ and $f: R \rightarrow B$ is locally holomorphic.

We define

$$
\begin{aligned}
& S\left(k_{1}, \ldots, k_{r}, b_{1}, \ldots, b_{r}\right) \\
& \quad=\frac{1}{b_{1} \ldots b_{r} p_{1}^{k_{1}} \ldots p_{r}^{k_{r}}} \sum_{i=1}^{r} \sum_{n_{i}=0 ; n_{i} \equiv a_{i}\left(\bmod M_{i}\right)}^{M_{i} b_{i} p^{k_{i}-1}} f\left(n_{1}, \ldots, n_{r}\right) .
\end{aligned}
$$

Then

(i) $L=\lim _{\left(k_{1}\right\} \rightarrow \infty} S\left(k_{1}, \ldots, b_{r}\right)$ exists;

(ii) $L$ is independent of the $\left\{b_{i}\right\}$ used;

(iii) $L$ may be calculated by iteration of the limit in any order.

Proof. We begin with $a_{1}, \ldots, a_{r}=0, M_{1}, \ldots, M_{r}=1$ and $f$ holomorphic on $R$ with $Z_{p}^{r} \subset R$. We can write

$$
f(\mathbf{x})=\sum_{J} a_{J} \mathbf{x}^{J}
$$

where the right side represents a power series in $r$ variables with $J$ running through the $r$-tuples of nonnegative integers.

After we substitute the series for $f$ in the formula for $S$ and use the fact (see [2]) that

$$
\left|(-1)^{j} B_{j}-\frac{1}{b p^{k}} \sum_{n=0}^{b p^{k}-1} n^{j}\right|_{p}<p^{2-k}
$$

for $k=0,1, \ldots$ and $j=0,1, \ldots$, it is easy to verify that 


$$
\lim _{k_{r} \rightarrow \infty} \ldots \lim _{k_{1} \rightarrow \infty} S\left(k_{1}, \ldots, b_{r}\right)=\sum_{J} a_{J}(-1)^{J} B_{J}
$$

and each limit is uniform with respect to the remaining variables. We can now conclude that

$$
L=\lim _{\left\{k_{i}\right\} \rightarrow \infty} S\left(k_{1}, \ldots, b_{r}\right) \text { exists. }
$$

The next two parts of the theorem are obvious.

Now suppose $f$ is locally holomorphic on $R$ and $R \supset Z_{p}^{r}$. Then there is a finite covering of $Z_{p}^{r}$ by polydiscs whereby $f$ is holomorphic on each polydisc, each polydisc has the same radius and the radius has the form $\left(p^{-N}, \ldots, p^{-N}\right)$. We let $A=\left\{0, \ldots, p^{N}-1\right\}$ and for each $W \in A^{r}$ we define

$$
f_{W}(\mathbf{x})=p^{-N r} f\left(W+p^{N} \mathbf{x}\right) .
$$

Each $f_{W}$ is holomorphic on the disc with center $(0, \ldots, 0)$ and radius $(1, \ldots, 1)$.

It is convenient now to introduce an integral type notation.

We define

$$
\int_{\mathbf{a}, \mathbf{M}} f(\mathbf{x}) d \mathbf{x}=L
$$

where $L$ is defined in Theorem 1 .

We have

$$
\int_{0,1} f(\mathbf{x}) d \mathbf{x}=\sum_{W \in A^{r}} \int_{0,1} f_{W}(\mathbf{x}) d \mathbf{x}
$$

and the conclusion of the theorem follows directly.

Finally, if $f: R \rightarrow B$ is locally holomorphic and $\mathbf{a}+\mathbf{M} Z_{p} \subset R$, we define $g(\mathbf{x})=f\left(\mathbf{a}^{\prime}+\mathbf{x M}\right)$ where $\mathbf{x M}=\left(x_{1} M_{1}, \ldots, x_{r} M_{r}\right)$ and $\mathbf{a}^{\prime}$ is the least nonnegative residue of $a \bmod M$. Since $g$ satisfies the conditions needed earlier in this proof, and $\int_{\mathrm{a}, \mathbf{M}} f(\mathbf{x}) d \mathbf{x}=\int_{0,1} g(\mathbf{x}) d \mathbf{x}$, we have established Theorem 1.

The next result is our basic device for constructing $p$-adic functions. We will use it to define a $p$-adic analog of $\log \Gamma$ and to define $p$-adic Euler constants. It can be used to show the existence and holomorphy of the $p$-adic $L$-functions and similarly constructed functions occurring in the works cited as references.

TheOREM 2. Suppose $a_{i}, M_{i}$ are rational integers with $a_{i}>0, M_{i}>1$ for $i=1,2, \ldots, r,\left\{C_{1}, \ldots, C_{t}\right\}$ is a set of polydiscs in $\Omega_{p}^{r}, R=\cup_{i=1}^{t} C_{i}$ and $\mathbf{a}+\mathbf{M} Z_{p} \subset R$. Let $\dot{D}$ be a polydisc in $\Omega_{p}^{s}$ and suppose $f: R \times D \rightarrow \Omega_{p}$ is holomorphic on each $C_{i} \times D, i=1,2, \ldots, t$. Then 


$$
F(\mathbf{x})=\int_{\mathbf{a}, \mathbf{M}} f(\mathbf{u}, \mathbf{x}) d \mathbf{u}
$$

exists and is holomorphic on $D$.

Proof. We let $\Lambda(D)=$ Banach space of holomorphic functions from $D \rightarrow \Omega_{p}$. For $u \in R$ we define

$$
\phi(\mathbf{u})=\text { the mapping } \mathbf{x} \rightarrow f(\mathbf{u}, \mathbf{x}) .
$$

For a fixed $\mathbf{u}_{i} \in C_{i}$ we have

$$
f(\mathbf{u}, \mathbf{x})=\sum_{J} a_{i, J}(\mathbf{x})\left(\mathbf{u}-\mathbf{u}_{i}\right)^{J}
$$

for all $\mathbf{u} \in C_{i}$ and $\mathbf{x} \in D$.

If $a_{i, J}$ denotes the map on $D, \mathbf{x} \rightarrow a_{i, J}(\mathbf{x})$, then

$$
\phi(\mathbf{u})=\sum_{J} a_{i, J}\left(\mathbf{u}-\mathbf{u}_{i}\right)^{J}
$$

for $\mathbf{u} \in C_{i}$.

Each $a_{i, J} \in \Lambda(D)$, so $\phi: R \rightarrow \Lambda(D)$ is locally holomorphic and we may apply Theorem 1 . Since

$$
\int_{\mathbf{a}, \mathbf{M}} f(\mathbf{u}, \mathbf{x}) d \mathbf{u}=\left(\int_{\mathbf{a}, \mathbf{M}} \phi(\mathbf{u}) d \mathbf{u}\right)(x)
$$

we conclude that $F \in \Lambda(D)$.

The following corollary is a useful form of Theorem 2.

COROLlaRY. Suppose $a, b, M$ are rational integers with $a>0, b>1, M>$ 1. Let $f$ be locally holomorphic on a set $A \subset \Omega_{p}$. Let $\mathrm{x} \in \Omega_{p}^{s}$ and $T(u, \mathrm{x})$ be locally holomorphic on some subset of $\Omega_{p}^{s+1}$. Define $A^{*}=\left\{\mathbf{x} \mid T\left(a+M Z_{p}, \mathbf{x}\right) \subset\right.$ A\}.

Then $A^{*}$ is open, and if $A^{*} \neq \varnothing$,

$$
F(\mathbf{x})=\lim _{k \rightarrow \infty} \frac{1}{b p^{k}} \sum_{\substack{n=0 \\ n \equiv a(\bmod M)}}^{M b p^{k}-1} f(T(n, \mathbf{x}))
$$

is independent of $b$ and locally holomorphic on $A^{*}$.

Proof. Given $c \in A^{*}$ and $u \in a+M Z_{p}$ there is a polydisc $D(u, c)$ containing $(u, c)$ on which $f \circ T$ is holomorphic. Holding $\mathrm{c}$ fixed, a finite number of $D\left(u_{i}, \mathrm{c}\right)$ cover $\left(a+M Z_{p}, \mathrm{c}\right)$. Each $D\left(u_{i}, \mathrm{c}\right)$ has the form $C_{i} \times D_{i}$ where $C_{i}$ is a disc in $\Omega_{p}$ containing $u_{i}$ and $D_{i}$ is a polydisc about c. Let $D=\cap D_{i}$. We know the following:

(i) $T\left(C_{i} \times D\right) \subset A$ for each $i$, so $D \subset A^{*}$;

(ii) $\cup C_{i}$ covers $a+M Z_{p}$;

(iii) $f \circ T$ is holomorphic on each $C_{i} \times D$. 
From Theorem 2 we see $F(x)$ is holomorphic on $D$ and therefore locally holomorphic on $A^{*}$.

We will occasionally wish to differentiate $F(\mathbf{x})$. We have

TheOREM 3. Using the definitions and conditions of Theorem 2,

$$
\frac{\partial F(\mathbf{x})}{\partial x_{i}}=\int_{\mathbf{a}, \mathbf{M}} \frac{\partial f(\mathbf{u}, \mathbf{x})}{\partial x_{i}} d \mathbf{u} \text {. }
$$

Proor. We fix $x \in D$ and for $t \in \Omega_{p}$ we let $t^{*}=(0, \ldots, t, \ldots, 0), t$ being in the $i$ th position, $t^{*} \in \Omega_{p}^{s}$. We define

$$
h(\mathbf{u}, t)=\frac{f\left(\mathbf{u}, \mathbf{x}+t^{*}\right)-f(u, x)}{t} \text { for } t \neq 0
$$

and $h(u, 0)=\lim _{t \rightarrow 0} h(u, t)$.

We observe that $h(\mathbf{u}, 0)=\partial f(\mathbf{u}, \mathbf{x}) / \partial x_{i}$ and that there is a neighborhood $D_{0}$, of zero, so $h$ is holomorphic on each $C_{i} \times D_{0}$.

From the definition of derivative we have

$$
\frac{\partial F(\mathbf{x})}{\partial x_{i}}=\lim _{t \rightarrow 0} \int_{\mathbf{a}, \mathbf{M}} h(\mathbf{u}, t) d \mathbf{u} \text {. }
$$

Now, if we let $H(t)=\int_{a, M} h(\mathrm{u}, t) d \mathrm{u}$ we can use Theorem 2 to see that $H$ is holomorphic on $D_{0}$ and, in particular, continuous at 0 .

Thus we have

$$
\frac{\partial F(\mathbf{x})}{\partial x_{i}}=\lim _{t \rightarrow 0} H(t)=H(0)=\int_{a, M} \frac{\partial f(\mathbf{u}, \mathbf{x})}{\partial x_{i}} d u .
$$

The next result shows how certain sums can be used to solve difference equations.

THEOREM 4. If $a, M$ are rational integers where $M>a>0, f^{\prime}(x+a)$ exists and $F(x)=\int_{a, M} f(x+u) d u$, then $F(x+M)$ exists and $F(x+M)=F(x)$ $+M f^{\prime}(x+a)$.

Proof. This follows directly from the definition of the right side.

4. The $p$-adic $\log \Gamma$ function. We now consider the problem of constructing a $p$-adic analog of $\log \Gamma(x)$.

In looking for a $p$-adic analog of $\log \Gamma(x)$ we want a function $G_{p}$ which sends a subset of $\Omega_{p}$ into $\Omega_{p}$ and satisfies the functional equation $G_{p}(x+1)$ $=G_{p}(x)+\log (x) \cdot \log (x)$ is defined by the usual power series when $|x-1|_{p}$ $<1$, and by setting $\log (p)=0$ and using the functional equations for $\log (x)$ when $|x-1|_{p}>1$ and $x \neq 0$. There is a complete discussion of this idea in [3]. Just as in the complex case, this functional equation forces $G_{p}$ to be discontinuous on either the positive integers or the negative integers. This is somewhat unfortunate in the $p$-adic case because if we want a locally 
holomorphic function we must exclude $Z_{p}$ from the domain of $G_{p}$. However, this is all we need exclude because on the domain $\Omega_{p}-Z_{p}$ we have a locally holomorphic function which satisfies $G_{p}(x+1)=G_{p}(x)+\log (x)$ and several other relations similar to those of the complex $\log \Gamma(x)$. We will use the construction given in $\$ 3$ to define $G_{p}(x)$ and demonstrate its properties.

An alternative approach is to slightly modify the functional equation to obtain a functional locally holomorphic on all of $\Omega_{p}$. After considering $G_{p}(x)$ we will exhibit a sequence of such functions. We have the relation that the sequence of functions locally holomorphic on $\Omega_{p}$ converges pointwise to $G_{p}$.

The technique of changing the functional equation has been used by Morita [7] to define $\Gamma_{p}$, a function on $Z_{p}$, which is an analog of $\Gamma$. Our $G_{p}$ is clearly not $\log \Gamma_{p}$.

DEFINITION OF $G_{p}$. We use the corollary of Theorem 2 with $T(u, x)=u+$ $x$ and $f(x)=x \log (x)-x . f$ is locally holomorphic on $\Omega_{p}-\{0\}$. We then have

$$
G_{p}(x)=\lim _{k \rightarrow \infty} \frac{1}{p^{k}} \sum_{n=0}^{p^{k}-1}(x+n) \log (x+n)-(x+n) .
$$

$G_{p}$ is locally holomorphic on $\Omega_{p}-Z_{p}$, and at each $c \in \Omega_{p}-Z_{p}$ the disc of holomorphy is the largest (open) disc $D(c)$ such that $D(c) \cap Z_{p}=\varnothing$.

An immediate consequence of Theorem 4 is the functional equation:

Theorem 5. $G_{p}(x+1)=G_{p}(x)+\log x$.

Stirling's Theorem, which is an asymptotic formula for $\log \Gamma(x)$, is simpler in $\Omega_{p}$. We have

THEOREM 6. When $|x|_{p}>1$,

$$
G_{p}(x)=\left(x-\frac{1}{2}\right) \log (x)-x+\sum_{r=1}^{\infty} \frac{B_{r+1}}{r(r+1) x^{r}} .
$$

Proof.

$$
G_{p}(x)=\frac{1}{2}-x+\lim _{k \rightarrow \infty} \frac{1}{p^{k}} \sum_{n=0}^{p^{k}-1}(x+n)(\log (x)+\log (1+n / x)) .
$$

Using the power series for $\log (1+n / x)$ will lead to the result.

If we match Theorem 6 and the next result with the corresponding classical formulas we see that it is more accurate to speak of $G_{p}(x)$ as the analog of $-\frac{1}{2} \log (2 \pi)+\log \Gamma(x)$. However, for simplicity we will continue to refer to $G_{p}$ as the analog of $\log \Gamma$.

The following relation is the $p$-adic version of Gauss' Multiplication Theorem.

THEOREM 7. Given any $m \in Z^{+}$we have 


$$
G_{p}(x)=\left(x-\frac{1}{2}\right) \log (m)+\sum_{a=0}^{m-1} G_{p}\left(\frac{x+a}{m}\right)
$$

provided the right side is defined.

Proof. We can write

$$
\begin{aligned}
& G_{p}(x)=\lim _{k \rightarrow \infty} \frac{1}{m p^{k}} \sum_{n=0}^{m p^{k}-1}(x+n) \log (x+n)-(x+n) \\
& =\lim _{k \rightarrow \infty} \frac{1}{m p^{k}} \sum_{n=0}^{p^{k}-1} \sum_{a=1}^{m-1}(x+a+m n) \log (x+a+m n)-(x+a+m n) .
\end{aligned}
$$

With a little rearranging, Theorem 7 is easily obtained.

COROllary.

$$
G_{p}(x)=\sum_{a=0}^{p^{r}-1} G_{p}\left(\frac{x+a}{p^{r}}\right) \text { for } r=0,1,2, \ldots
$$

This last corollary provides us with a means for transferring results about $G_{p}(x)$ when $|x|_{p}>1$ to $G_{p}(x)$ with $|x|_{p}<1$.

For the extension theorem we have

TheOREM 8. $G_{p}(x)+G_{p}(1-x)=0$.

Proof. We can see immediately from Theorem 6 that $G_{p}(x)+G_{p}(-x)=$ $-\log (x)$ when $|x|_{p}>1$. Combining this with $G_{p}(x+1)=G_{p}(x)+\log (x)$ we have $G_{p}(x)+G_{p}(1-x)=0$ when $|x|_{p}>1$.

Given any $x \in \Omega_{p}-Z_{p}$ with $|x|_{p}<1$ we can choose an $r \in Z^{+}$so $\left|(x+a) / p^{r}\right|_{p}>1$ for all $a \in Z$. Then

$$
\begin{aligned}
G_{p}(x)+G_{p}(1-x) & =\sum_{a=0}^{p^{r}-1} G_{p}\left(\frac{x+a}{p^{r}}\right)+G_{p}\left(\frac{1-x+a}{p^{r}}\right) \\
& =\sum_{a=0}^{p^{r}-1} G_{p}\left(\frac{x+a}{p^{r}}\right)-G_{p}\left(1-\frac{1-x+a}{p^{r}}\right) \\
& =\sum_{a=0}^{p^{r}-1} G_{p}\left(\frac{x+a}{p^{r}}\right)-G_{p}\left(\frac{x+p^{r}-a-1}{p^{r}}\right),
\end{aligned}
$$

and, since as $a$ goes from 0 to $p^{r}-1, p^{r}-a-1$ goes from $p^{r}-1$ to 0 , Theorem 8 is proven.

The complex $\log \Gamma(x)$ has a simple power series about 1 , with values of the Riemann $\zeta$-function appearing in the coefficients. We will now find the power series for $G_{p}(x)$ about $1 / p$.

We use Theorem 4 to obtain 


$$
D^{(1)} G_{p}(x)=\lim _{k \rightarrow \infty} \frac{1}{p^{k}} \sum_{n=0}^{p^{k}-1} \log (x+n)
$$

and, in particular,

$$
D^{(1)} G_{p}(1 / p)=p \lim _{k \rightarrow \infty} \frac{1}{p^{k}} \sum_{\substack{m=0 \\ m \equiv 1(\bmod p)}}^{p^{k}-1} \log (m)
$$

We write this as

$$
\begin{aligned}
D^{(1)} G_{p}(1 / p) & =-p \gamma_{p}(1, p), \\
D^{(r)} G_{p}(x) & =(-1)^{r}(r-2) ! \lim _{k \rightarrow \infty} \frac{1}{p^{k}} \sum_{n=0}^{p^{k}-1} \frac{1}{(x+n)^{r-1}} \quad \text { for } r \geqslant 2, \\
\frac{D^{(r)} G_{p}(1 / p)}{r !} & =\frac{(-1)^{r}}{r(r-1)} \lim _{k \rightarrow \infty} \frac{1}{p^{k}} \sum_{n=0}^{p^{k}-1} \frac{1}{(n+1 / p)^{r-1}} \\
& =\frac{(-1)^{r} p^{r}}{r} \cdot \frac{1}{r-1} \lim _{k \rightarrow \infty} \frac{1}{p^{k}} \sum_{\substack{m=0 \\
m \equiv 1(\bmod p)}}^{p^{k}-1} \frac{1}{m^{r-1}} .
\end{aligned}
$$

We will write this last expression as $(-1)^{r} p \zeta_{p}(r) / r$. Using the notation introduced above we have

THEOREM 9.

$$
G_{p}(x)=G_{p}\left(\frac{1}{p}\right)-p \gamma_{p}(1, p)\left(x-\frac{1}{p}\right)+\sum_{r=2}^{\infty} \frac{(-1)^{r} \zeta_{p}(r) p^{r}}{r}\left(x-\frac{1}{p}\right)^{r} \text {. }
$$

This series converges for $|x-1 / p|_{p}<p$.

It is interesting to compare this with the classical formula for $\log \Gamma(x)$ :

$$
\log \Gamma(x)=-\gamma(x-1)+\sum_{r=2}^{\infty} \frac{(-1)^{r} \zeta(r)}{r}(x-1)^{r} \text { for }|x-1|<1 .
$$

The idea of having $p=1$ give us classical results from a $p$-adic formula, while only formal here, is valid in certain formulas for $\zeta(n)$ and $\zeta_{p}(n)$ discussed in [1].

Our next result for $G_{p}(x)$ is a set of formulas for $G_{p}(x)$ valid on the annular regions $A_{n}=\{x: n-1<\nu(x)<n\}$ for $n=1,2,3, \ldots$. Since these regions have no points of $Z_{p}$ we are able to find series which are almost Laurent series. To simplify the discussion we introduce a function $G^{*}$ defined by

$$
G^{*}(x)=\lim _{k \rightarrow \infty} \frac{1}{p^{k}} \sum_{\substack{n=0 \\ p \nmid n}}^{p^{k}-1} f(x+n)
$$

where $f(x)=x \log (x)-x$ 
We can write

$$
G^{*}(x)=\sum_{a=1}^{p-1} \lim _{k \rightarrow \infty} \frac{1}{p^{k}} \sum_{\substack{n=0 \\ n \equiv a(\bmod p)}}^{p^{k}-1} f(x+n) .
$$

For each value of $a$ the inner lim is locally analytic for $x$ with $x+a \notin p Z_{p}$. Therefore $G^{*}$ is locally analytic for $x \in \Omega_{p}-V_{p}$, where $V_{p}$ is the set of units in $Z_{p}$.

For $|x|_{p}<1, G^{*}$ coincides with a function defined by Morita [7] in the study of the function he calls $\Gamma_{p}(x)$.

To obtain our formulas for $G_{p}$ we need the power series for $G^{*}(x)$ at $x=0$.

THEOREM 10. If $|x|_{p}<1$, then

$$
G^{*}(x)=M_{\varepsilon}(\log )(x)+\sum_{r=3}^{\infty} \frac{L_{p}\left(r, \bar{\Phi}^{r-1}\right)(-1)^{r}}{r} x^{r} .
$$

$L_{p}(r, \chi)$ is the Leopoldt L-function for the character $\chi, \varepsilon_{p}$ is the principal character $\bmod p, M_{\chi}(f)$ is the Leopoldt $\chi$-mean [5], and $\frac{p}{\omega}$ is the character $\bmod p$ defined by

$$
\bar{\omega}(n)=\left\{\begin{array}{l}
\lim _{k \rightarrow \infty} n^{-p^{k}} \text { for }(n, p)=1, \text { if } p>2, \\
1 \quad \text { if } n \equiv 1 \bmod 4, \\
-1 \quad \text { if } n \equiv 3 \bmod 4 \text { for } p=2 .
\end{array}\right.
$$

Proof. Apply Theorem 3.

This result has also been found by Morita [7].

We are now prepared to find series for $G_{p}(x)$ on the annular domains $A_{n}=\{x: n-1<\nu(x)<n\}, n \in Z^{+}$.

With $x \in A_{n}$ we write the equations

$$
G_{p}\left(x / p^{i}\right)-G_{p}\left(x / p^{i+1}\right)=G^{*}\left(x / p^{i}\right) \text { for } i=0,1, \ldots, n-1 \text {; }
$$

adding these equations we obtain

$$
G_{p}(x)=G_{p}\left(\frac{x}{p^{n}}\right)+\sum_{i=0}^{n-1} G^{*}\left(\frac{x}{p^{i}}\right) .
$$

We now use Theorems 6 and 10 to obtain

THEOREM 11. On the annulus $A_{n}$ we have the formula

$$
\begin{aligned}
G_{p}(x)= & \left(\frac{x}{p^{n}}-\frac{1}{2}\right) \log (x)-\frac{x}{p^{n}}+M_{p}(\log )\left(\frac{p^{n}-1}{p^{n}-p^{n-1}}\right) x \\
& +\sum_{r=3}^{\infty} \frac{L_{p}\left(r, \bar{\omega}^{r-1}\right)(-1)^{r}}{r}\left(\frac{p^{r n}-1}{p^{r n}-p^{r(n-1)}}\right) x^{r}+\sum_{r=1}^{\infty} \frac{B_{r+1} p^{n r} x^{-r}}{r(r+1)} .
\end{aligned}
$$


If we define $A_{0}$ as $\left\{x:|x|_{p}>1\right\}$, then the above formula is valid for $n=$ $0,1,2, \ldots$

5. Analyticity. The function $G_{p}$ is not an analytic functionin the sense of Krasner [4], but its second derivative $G_{p}^{\prime \prime}$ is an analytic function on $\Omega_{p}-Z_{p}$.

THEOREM 12. $G_{p}^{\prime \prime}$ is an analytic function on $\Omega_{p}-Z_{p}$.

Proof. First for $a, m \in Z$ we define $D(a, m)=\left\{x: x \in \Omega_{p}, \nu(x-a)>\right.$ $m$ \}. For each $m \in Z^{+}$the set $A_{m}=\Omega_{p}-\cup_{a=0}^{p^{n}-1} D(a, m)$ is a quasi-connected set. $\left\{A_{m}: m \in Z^{+}\right\}$is nested and $\cup_{m=1}^{\infty} A_{m}=\Omega_{p}-Z_{p}$. Therefore if we can prove $G_{p}^{\prime \prime}$ is an analytic element on each $A_{m}$, i.e. the uniform limit of a sequence of rational functions having no poles in $A_{m}$, then we will know $G_{p}^{\prime \prime}$ is an analytic function on $\Omega_{p}-Z_{p}$.

If we apply Theorem 7 we can write

$$
G_{p}^{\prime \prime}(x)=\frac{1}{p^{2 m+2}} \sum_{a=0}^{p^{m+1}-1} G_{p}^{\prime \prime}\left(\frac{x+a}{p^{m+1}}\right)
$$

for each $m \in Z^{+}$and $x \in \Omega_{p}-Z_{p}$.

If we consider just $x \in A_{m}$, then $\left|(x+a) / p^{m+1}\right|_{p}>p>1$ for all $a \in$ $Z$. Therefore we may use Theorem 6 and obtain

$$
G_{p}^{\prime \prime}\left(\frac{x+a}{p^{m+1}}\right)=\sum_{r=0}^{\infty} \frac{B_{r}}{\left[(x+a) / p^{m+1}\right]^{r+1}} .
$$

Since $\left|(x+a) / p^{m+1}\right|_{p}>p$ for all $x \in A_{m}$, this last series converges uniformly on $A_{m}$.

Thus $G_{p}^{\prime \prime}$ is an analytic element on $A_{m}$ and $G_{p}^{\prime \prime}$ is an analytic function on $\Omega_{p}-Z_{p}$.

6. An alternative approach. Earlier we mentioned another approach to the idea of $p$-adic $\log \Gamma(x)$ : to change the functional equation. Of course, it must only be a slight change so we can associate it with $\log \Gamma(x)$. We will construct a sequence of such functions, which will be locally holomorphic on $\Omega_{p}$ and have $G_{p}(x)$ as their pointwise limit.

DeFINITION. Let

$$
H_{N}(x)=\lim _{k \rightarrow \infty} \frac{1}{p^{k}} \sum_{n=0}^{p^{k}-1} f_{N}(x+n) \text { for } N=1,2, \ldots,
$$

where

$$
f_{N}(x)= \begin{cases}x \log (x)-x & \text { if } \nu(x)<N \\ 0 & \text { if } \nu(x)>N\end{cases}
$$

Each $f_{N}$ is locally analytic on $\Omega_{p}$, so each $H_{N}$ is also locally analytic on $\Omega_{p}$. 
We have the following equation

$$
H_{N}(x+1)= \begin{cases}H_{N}(x)+\log (x) & \text { if } \nu(x)<N \\ H_{N}(x) & \text { if } \nu(x)>N\end{cases}
$$

It can be shown that $H_{N}(0)=0$ for each $N$, so we have for $n \in Z^{+}$,

$$
H_{N}(n+1)=\log \prod_{\substack{t=1 \\ p^{N} \nmid t}}^{n} t
$$

in particular, $H_{N}(n+1)=\log (n !)$ if $n<p^{N}$. The following theorem shows the relation between $G_{p}(x)$ and $H_{N}(x)$.

THEOREM 13. If $x$ is such that $|x-a|_{p}>p^{-N}$ for all $a \in Z_{p}$ then $H_{N}(x)=$ $G_{p}(x)$.

Proof. Inspection of the definitions of $G_{p}(x)$ and $H_{N}(x)$.

Theorem 13 shows us that the sequence $H_{N}(x), N=1,2, \ldots$, and $x$ fixed with $x \notin Z_{p}$, eventually becomes constant with the value $G_{p}(x)$.

For $x$ with $\nu(x) \geqslant 1$ the functions $H_{1}$ and $G^{*}$ coincide. However, for other $x$ they are not the same and it is $H_{1}$ which is the log of the function on $Z_{p}$ which Morita has called $\Gamma_{p}(x)[7]$.

7. $p$-adic Euler constants. In a recent paper [6], D. H. Lehmer proves a theorem of Gauss by defining a generalization of Euler's constant. Gauss' theorem is a formula for the logarithmic derivative of the Gamma function at rational points $r / k$ with $0<r<k$. The formula is notable because it is a constant plus a linear combination of logarithms of integers in $Q(\sqrt[k]{1})$.

We shall define $p$-adic Euler constants, give the basic results for them and then prove the $p$-adic version of Gauss' theorem: a formula for $G_{p}^{\prime}(r / f)$ with $0<r<f$ and $\nu(r / f)<0$.

We show how Gauss' theorem follows from the classical formula for $L(1, \chi)$, and since we have the same expression for $L_{p}(1, \chi)$ [3], we have a proof valid in both $C$ and $\Omega_{p}$.

Lehmer defines the (generalized) Euler constants by

$$
\gamma(r, k)=\lim _{x \rightarrow \infty}\left[\sum_{\substack{0<n<x \\ n \equiv r(\bmod k)}} \frac{1}{n}-\frac{1}{k} \log x\right]
$$

and then, using the relation

$$
\psi(r / k)=D^{(1)} \log \Gamma(r / k)=\log k-k \gamma(r, k) \quad \text { for } r, k \in Z^{+}, r<k,
$$

he proves: If $r, k \in Z^{+}, r \leqslant k$, then 


$$
\begin{aligned}
\psi\left(\frac{r}{k}\right)= & -\gamma-\log \left(\frac{k}{2}\right)-\frac{\pi}{2} \cot \left(\frac{\pi r}{k}\right) \\
& +2 \sum_{0<j<k / 2} \cos \left(\frac{2 \pi r j}{k}\right) \log \sin \left(\frac{\pi j}{k}\right) .
\end{aligned}
$$

Gauss' theorem, combined with the functional equation, enables us to calculate $\psi(x)$ in closed form at every rational value of $x$ for which the function is defined.

Working in $\Omega_{p}$ we can define $\gamma_{p}(r, f)$ when $r, f \in Z, f>1$, and find a similar formula for $G_{p}^{\prime}(r / f)$.

When $\nu(r / f)<0$ we define

$$
\gamma_{p}(r, f)=-\lim _{k \rightarrow \infty} \frac{1}{f p^{k}} \sum_{\substack{m=0 \\ m \equiv r(\bmod f)}}^{f p^{k}-1} \log (m) .
$$

When $\nu(r / f) \geqslant 0$ we write $f=p^{k} f^{*}$ with $\left(p, f^{*}\right)=1$ and let $\phi=\phi\left(f^{*}\right)$ (the Euler function). We then define

$$
\gamma_{p}(r, f)=\frac{p^{\phi}}{p^{\phi}-1} \sum_{n \in N(r, f)} \gamma_{p}\left(r+n f, p^{\phi} f\right)
$$

where

$$
N(r, f)=\left\{n: 0 \leqslant n<p^{\phi}, n f+r \neq 0\left(\bmod p^{\phi+k}\right)\right\}
$$

Theorem 1 applies to show $\gamma_{p}(r, f)$ exists.

To obtain Gauss' theorem in $\Omega_{p}$ we need several results which are mostly the same as Lehmer has given for $C$. The proofs follow from the definition of $\gamma_{p}(r, f)$, previous results for $G_{p}^{\prime}(x)$ and Theorem 18. We will write $\psi_{p}(x)=$ $G_{p}^{\prime}(x)$.

THEOREM 14. (i) If $d \mid(r, f)$, then $f \gamma_{p}(r, f)=(f / d) \gamma_{p}(r / d, f / d)-\log d$.

(ii) If $\nu(r / f)<0$ and $0<r<f$, then $\psi_{p}(r / f)=-\log f-f \gamma_{p}(r, f)$.

(iii) $\gamma_{p}(r, f)=\gamma_{p}(f-r, f)$.

(iv) If $b \in Z^{+}$, then

$$
\gamma_{p}(r, f)=\sum_{n=0}^{b-1} \gamma_{p}(r+n f, b f) .
$$

(v) If $p^{\mu} \equiv 1\left(\bmod f^{*}\right)$ and $\nu(r / f)>0$, then

$$
\gamma_{p}(r, f)=\frac{p^{\mu}}{p^{\mu}-1} \sum_{\substack{n=0 \\ n f+r \neq 0\left(\bmod p^{\mu+k}\right)}}^{p^{\mu}-1} \gamma_{p}\left(r+n f, p^{\mu} f\right) .
$$

We are going to need a $p$-adic analog of Euler's constant. The value 


$$
\gamma_{p}=\gamma_{p}(0,1)=-\frac{p}{p-1} \lim _{k \rightarrow \infty} \frac{1}{p^{k}} \sum_{\substack{m=1 \\(m, p)=1}}^{p^{k}-1} \log (m)
$$

fits our formulas precisely as we need. Morita has also realized the connection between Euler's constant and $M_{\xi}(\log )$ and he gives a slightly different value [7].

Lehmer has defined the formula $\Phi(f)$ by

$$
\Phi(f)=\sum_{\substack{r=1 \\(r, f)=1}}^{f} \gamma(r, f) .
$$

He then proves

$$
\Phi(f)=\frac{\phi(f)}{f} \gamma+\frac{\phi(f)}{f} \sum_{q \mid f} \frac{\log q}{q-1} .
$$

In this formula, $q$ is prime, $\phi$ is the Euler $\phi$-function and $\gamma$ is Euler's constant. We define

$$
\Phi_{p}(f)=\sum_{\substack{r=1 \\(r, f)=1}}^{f} \gamma_{p}(r, f) \quad \text { when } \nu(f)>0 .
$$

We then have

THEOREM 15.

$$
\Phi_{p}(f)=\frac{\phi(f)}{f} \gamma_{p}+\frac{\phi(f)}{f} \sum_{q \mid f} \frac{\log q}{q-1} .
$$

Proof. We can use Theorem 14(iv) with $b=f_{1} / f$ to show that if $f$ has the same distinct prime factors as $f_{1}$ and $f \mid f_{1}$ then $\Phi_{p}(f)=\Phi_{p}\left(f_{1}\right)$.

It is then sufficient to consider square free $f$. This is accomplished by induction on the number of prime factors of $f$.

We will need the following algebraic identity.

THEOREM 16. If $\zeta$ is a primitive fth root of unity, $f>1, \varepsilon_{f}$ the principal character $\bmod f$ and $\tau_{a}\left(\varepsilon_{f}\right)$ the Gauss sum,

$$
\tau_{a}\left(\varepsilon_{f}\right)=\sum_{n=1}^{f} \varepsilon_{f}(n) \zeta^{a n}
$$

then

$$
\prod_{a=1}^{f-1}\left(1-\zeta^{-a}\right)^{\tau_{a}\left(\varepsilon_{f}\right)}=\prod_{q \mid f} q^{-\phi(f) /(q-1)} .
$$

The product on the right side is over the distinct prime divisors of $f$. 
PROOF. Let $\omega_{r}$ be a primitive $r$ th root of unity and $Q_{r}=Q\left(\omega_{r}\right)$ for $r=2,3, \ldots$.

We observe that

$$
\tau_{a}\left(\varepsilon_{f}\right)=\sum_{\substack{n=1 \\(n, f)=1}}^{f-1} \zeta^{a n}=\operatorname{tr}_{Q / Q}\left(\zeta^{a}\right) .
$$

When we group together conjugate elements we have

$$
\prod_{a=1}^{f-1}\left(1-\zeta^{-a}\right)^{\tau_{a}\left(\xi_{j}\right)}=\prod_{r \mid f}\left(N_{Q / Q}\left(1-\omega_{r}\right)\right)^{\mathrm{tr}_{Q_{f / Q}}\left(\omega_{r}\right)} .
$$

Examination of the minimal polynomial of $\omega_{r}$ shows that:

(i) if $r$ is not square free, then $\operatorname{tr}_{Q, / Q}\left(\omega_{r}\right)=0$;

(ii) if $r$ is square free, but not prime, then $N_{Q_{r} / Q}\left(1-\omega_{r}\right)=1$;

(iii) if $r$ is prime, $N_{Q / Q}\left(1-\omega_{r}\right)=r$;

(iv) if $r$ is prime

$$
\operatorname{tr}_{Q_{f} / Q}\left(\omega_{r}\right)=\frac{\phi(f)}{r-1} \operatorname{tr}_{Q_{r} / Q}\left(\omega_{r}\right)=\frac{-\phi(f)}{r-1} .
$$

Placing these four values into (1) establishes the theorem.

Now we state Gauss' theorem in $\Omega_{p}$.

THEOREM 17. If $r, f \in Z^{+}, r<f$ and $\nu(r / f)<0$, then

$$
\psi_{p}(r / f)=-\log f-\gamma_{p}+\sum_{a=1}^{f-1} \zeta^{-a r} \log \left(1-\zeta^{a}\right) .
$$

If $\psi_{p}$ is replaced by $\psi$ and $\gamma_{p}$ by $\gamma$ we have Gauss' theorem in $C$. Of course $\log$ is either $p$-adic or complex as required.

Proof. Since we have shown (Theorem 14(ii)) that $\psi_{p}(r / f)=-\log f-$ $f \gamma_{p}(r, f)$, it will be sufficient to prove

THEOREM 18. If $f>1$ and $\zeta$ is a primitive fth root of unity, then

$$
f \gamma_{p}(r, f)=\gamma_{p}-\sum_{a=1}^{f-1} \zeta^{-a r} \log \left(1-\zeta^{a}\right) .
$$

Notice that we do not need the restriction $\nu(r / f)<0$ for this result.

Proof. We begin by assuming $(r, f)=1$ and $\nu(r / f)<0$ and proceed to evaluate $\Sigma_{\chi \neq \varphi} \bar{\chi}(r) L_{p}(1, \chi)$ in two different ways. The sum is over all nonprincipal characters $\bmod f$.

For $\chi$ not principal and if $p \mid f$, we have the forumla [3]

$$
L_{p}(1, \chi)=-\frac{1}{f} \sum_{a=1}^{f-1} \tau_{a}(\chi) \log \left(1-\zeta^{-a}\right) .
$$


(NoTE: Iwasawa gives this formula in a form valid only for primitive characters, but if $\bar{\chi}(a) \tau(\chi)$ is replaced by $\tau_{a}(\chi)$, then his proof can be modified to be valid for all nonprincipal characters.)

This is the same as the formula for $L(1, \chi)$ in $C$. Using this result and Theorems 15 and 16 we have

(*) $\sum_{\chi \neq \varepsilon_{j}} \bar{\chi}(r) L_{p}(1, \chi)=\frac{\phi(f) \gamma_{p}}{f}-\Phi_{p}(f)-\frac{\phi(f)}{f} \sum_{a=1}^{f-1} \zeta^{a r} \log \left(1-\zeta^{-a}\right)$.

This result is also valid, correctly interpreted, in $C$.

If we use the expression [5] that

$$
L_{p}(1, \chi)=-\lim _{k \rightarrow \infty} \frac{1}{f p^{k}} \sum_{n=0}^{f p^{k}-1} \chi(n) \log n \quad \text { where }(n, p)=1,
$$

we obtain

(**)

$$
\sum_{\chi \neq \varepsilon_{f}} \bar{\chi}(r) L_{p}(1, \chi)=\phi(f) \gamma_{p}(r, f)-\Phi_{p}(f) .
$$

(**) is obtained in $C$ by using

$$
L(1, \chi)=\lim _{x \rightarrow \infty} \sum_{0<n<x} \frac{\chi(n)}{n} .
$$

Equating (*) and (**) yields Theorem 18 in the case where $(r, f)=1$ and $\nu(r / f)<0$.

Now suppose $(r, f)=d>1$ and $\nu(r / f)<0$. Then we can use 14(i) to obtain

$$
f \gamma_{p}(r, f)=-\log d+\gamma_{p}-\sum_{a=1}^{f / d-1} \zeta^{-a r} \log \left(1-\zeta^{a d}\right) .
$$

We can factor $1-\zeta^{a d}$ and obtain

$$
f \gamma_{p}(r, f)=-\log d+\gamma_{p}-\sum_{a=1}^{f / d-1} \sum_{b=0}^{d-1}\left(\zeta^{a} \lambda^{b}\right)^{-r} \log \left(1-\zeta^{a} \lambda^{b}\right)
$$

where $\lambda$ is a primitive $d$ th root of unity.

Since

$$
\begin{aligned}
\left\{\zeta^{a} \lambda^{b}\right. & 0<a<f / d, 0<b \leqslant d-1\}=\left\{\zeta^{a}: 0 \leqslant a<f\right\}, \\
f \gamma_{p}(r, f) & =-\log d+\gamma_{p}-\sum_{a=1}^{f-1} \zeta^{-a r} \log \left(1-\zeta^{a}\right)+\sum_{b=1}^{d-1} \log \left(1-\lambda^{b}\right) \\
& =\gamma_{p}-\sum_{a=1}^{f-1} \zeta^{-a r} \log \left(1-\zeta^{a}\right) .
\end{aligned}
$$

This completes the proof of Gauss' theorem, but we have not yet completed the proof of Theorem 18. 
If $\nu(r, f)>0$ we can use the definition of $\gamma_{p}(r, f)$ and the case of Theorem 18 already proven to show

$$
f \gamma_{p}(r, f)=\gamma_{p}-\frac{1}{p^{\phi}-1} \sum_{a=1}^{p^{\phi f-1}} \eta^{-a r} \log \left(1-\eta^{a}\right) \sum_{n \in N(r, f)} \eta^{-a n f},
$$

where $\eta$ is a primitive $p^{\phi} f$ root of unity.

The last sum on the right is $p^{\phi}-1$ if $p^{\phi} \mid a$ and $-\eta^{a r-a r p^{\phi}}$ if $p^{\phi} \nmid a$. Thus

$$
f \gamma_{p}(r, f)=\gamma_{p}-\sum_{a=1}^{f-1} \zeta^{-a r} \log \left(1-\zeta^{a}\right)+\frac{1}{p^{\phi}-1} \sum_{\substack{a=1 \\ p^{\phi} \nmid a}}^{p^{\phi}-1} \zeta^{-a r} \log \left(1-\eta^{a}\right) .
$$

The last sum on the right is 0 .

We have seen that $\psi_{p}$ is locally holomorphic on $\Omega_{p}-Z_{p}$ and $\psi_{p}^{\prime}$ is Krasner-analytic on this domain. We have also shown that the formula

$$
-\log f-f \gamma_{p}(r, f)
$$

depends only on the ratio $r / f$ and that for $\nu(r / f)<0$, and $0<r<f$,

$$
\psi_{p}(r / f)=(*)=-\log f-\gamma_{p}+\sum_{a=1}^{f-1} \zeta^{-a r} \log \left(1-\zeta^{a}\right) .
$$

Since (*) is defined for $r, f$ with $\nu(r / f)>0$, it is tempting to use (*) to extend the definition of $\psi_{p}$ onto the rational numbers in $Z_{p}$. However, this "continuation" would not retain the other properties of $\psi_{p}$. The values of (*), though, are related to functions similar to $\psi_{p}$ when $\nu(r, f)>0$ and we have

THEOREM 19. Given $\nu(r / f) \geqslant 0$, then for any $\mu$ such that $p^{\mu} \equiv 1\left(\bmod f^{*}\right)$ we have

$$
(*)=\frac{p^{\mu}}{p^{\mu}-1} H_{\mu}^{\prime}\left(\frac{r}{f}\right)=-\log f-\gamma_{p}+\sum_{a=1}^{f-1} \zeta^{-a r} \log \left(1-\zeta^{a}\right) .
$$

$H_{N}$ is discussed at the end of $\S 4$.

PROOF. This follows directly from previous results.

Since $H_{1}\left(\right.$ on $\left.Z_{p}\right)$ is the logarithm of Morita's $\Gamma_{p}[7]$, we have a

COROLLARY. If $0<r<f, v(r / f)>0$ and $f^{*} \mid(p-1)$, then

$$
\frac{\Gamma_{p}^{\prime}}{\Gamma_{p}}\left(\frac{r}{f}\right)=(1-1 / p)\left(-\log f-\gamma_{p}+\sum_{a=1}^{f-1} \zeta^{-a r} \log \left(1-\zeta^{a}\right)\right) .
$$

\section{REFERENCES}

1. J. Diamond, On the values of $p$-adic L-functions at positive integers (to appear).

2. J. Fresnel, Nombres de Bernoulli et fonctions $L$ p-adiques, Ann. Inst. Fourier (Grenoble) 17 (1967), fasc. 2, 281-333. (1968). MR 37 \# 169. 
3. K. Iwasawa, Lectures on p-adic L-functions, Ann. of Math. Studies, no. 74, Princeton Univ. Press, Princeton, N. J., 1972. MR 50 \# 12974.

4. M. Krasner, Rapport sur le prolongement analytique dans les corps valués complets par la méthode des éléments analytiques quasi-connexes, Table Ronde d'Analyse non archimédienne (Paris, 1972), Bull. Soc. Math. France, Mém. No. 39-40, Soc. Math. France, Paris, 1974, pp. 131-254. MR 52 \#6033.

5. T. Kubota and H. W. Leopoldt, Eine p-adishe Theorie der Zetawerte. I. J. Reine Angew. Math. 214/215 (1964), 328-339. MR 29 \#1199.

6. D. H. Lehmer, Euler constants for arithmetical progressions, Acta Arith. 27 (1975), 125-142. MR 51 \#5468.

7. Y. Morita, A p-adic analog of the $\Gamma$ function, (to appear).

8. N. Nielsen, Die Gammafunktion, Bände I, II, Chelsea, New York, 1965. MR 32 \#2622.

Department of Mathematics, Queens College, Flushing, New York 11367 